\title{
On non-linear action of multiple M2-branes
}

\author{
Mohammad R. Garousi \\ Department of Physics, Ferdowsi university, P.O. Box 1436, Mashhad, Iran \\ School of Physics, Institute for research in fundamental sciences (IPM), \\ P.O. Box 19395-5531, Tehran, Iran.
}

\begin{abstract}
A nonlinear $S O(8)$ invariant BF type Lagrangian for describing the dynamics of N M2branes in flat spacetime has been proposed recently in the literature which is an extension of the non-abelian DBI action of N D2-branes. This action includes only terms with even number of the totally antisymmetric tensor $M^{I J K}$. We argue that the action should contain terms with odd number of $M^{I J L}$ as well. We modify the action to include them.
\end{abstract}


Following the idea that the Chern-Simons gauge theory may be used to describe the dynamics of coincident M2-branes [1], Bagger and Lambert [2] as well as Gustavsson [3] have constructed three dimensional $\mathcal{N}=8$ superconformal $S O(4)$ Chern-Simons gauge theory based on 3-algebra. It is believed that the BLG world volume theory at level one describes two M2-branes on $R^{8} / Z_{2}$ orbifold [4]. The world volume theory of $\mathrm{N}$ M2-branes on $R^{8} / Z_{k}$ orbifold has been constructed in [5] which is given by $\mathcal{N}=6 U(N)_{k} \times U(N)_{-k}$ Chern-Simons gauge theory.

The signature of the metric on the 3 -algebra in the BLG model is positive definite. This assumption has been relaxed in [6] to study $N$ coincident M2-branes in flat spacetime. The so called BF membrane theory with arbitrary semi-simple Lie group has been proposed in [6]. This theory has ghost fields, however, there are different arguments that model may be unitary due to the particular form of the interactions [6, 7]. The bosonic part of the Lagrangian for gauge group $U(N)$ is given by 1

$$
L=\operatorname{Tr}\left(\frac{1}{2} \epsilon^{a b c} B_{a} F_{b c}-\frac{1}{2} \hat{D}_{a} X^{I} \hat{D}^{a} X^{I}+\frac{1}{12} M^{I J K} M^{I J K}\right)+\left(\partial_{a} X_{-}^{I}-\operatorname{Tr}\left(B_{a} X^{I}\right)\right) \partial^{a} X_{+}^{I}
$$

where $A_{a}, B_{a}, X^{I}$ are in adjoint representation of $U(N)$ and $X_{-}^{I}, X_{+}^{I}$ are singlet under $U(N)$, and

$$
\begin{aligned}
M^{I J K} & \equiv X_{+}^{I}\left[X^{J}, X^{K}\right]+X_{+}^{J}\left[X^{K}, X^{I}\right]+X_{+}^{K}\left[X^{I}, X^{J}\right] \\
\hat{D}_{a} X^{I} & =D_{a} X^{I}-X_{+}^{I} B_{a}, \quad D_{a} X^{I}=\partial_{a} X^{I}+i\left[A_{a}, X^{I}\right]
\end{aligned}
$$

Obviously the above Lagrangian is invariant under global $S O(8)$ transformation and under $U(N)$ gauge transformation associated with the $A_{a}$ gauge field. It is also invariant under gauge transformation associated with the $B_{a}$ gauge field

$$
\delta_{B} X^{I}=X_{+}^{I} \Lambda, \quad \delta_{B} B_{a}=D_{a} \Lambda, \quad \delta_{B} X_{+}^{I}=0, \quad \delta_{B} X_{-}^{I}=\operatorname{Tr}\left(X^{I} \Lambda\right)
$$

The Lagrangian (11) is a candidate to describe the dynamics of N M2-branes in flat supergravity background.

The equation of motion for $X_{-}^{I}$ gives $\partial_{a} \partial^{a} X_{+}^{I}=0$. Following the procedure found in [8], if one of the scalars $X_{+}^{I}$ takes large expectation value, i.e., $X_{+}^{I}=g_{Y M} \delta^{I 10}$, and the gauge symmetry (3) is fixed by setting $X^{10}=0$, then the above action reduces to

$$
L=\operatorname{Tr}\left(\frac{1}{2} \epsilon^{a b c} B_{a} F_{b c}-\frac{1}{2} D_{a} X^{i} D^{a} X^{i}-\frac{g_{Y M}^{2}}{2} B_{a} B^{a}-\frac{g_{Y M}^{2}}{4}\left[X^{i}, X^{j}\right]\left[X^{j}, X^{i}\right]\right)
$$

Under the dNS duality transformation [9]

$$
\operatorname{Tr}\left(\frac{1}{2} \epsilon^{a b c} B_{a} F_{b c}-\frac{g_{Y M}^{2}}{2} B_{a} B^{a}\right) \rightarrow \operatorname{Tr}\left(-\frac{1}{4 g_{Y M}^{2}} F^{a b} F_{a b}\right)
$$

\footnotetext{
${ }^{1}$ Our index convention is that $a, b, \ldots=0,1,2 ; i, j, \ldots=3, \ldots, 9$ and $I, J, \ldots=3,4, \ldots, 10$.
} 
the action becomes identical to the low energy action of N D2-branes in flat spacetime. The nonlinear extension of this action is given by the following nonabelian DBI action [10, 11]:

$$
\begin{aligned}
S^{D_{2}}= & -T_{2} \int d^{3} \sigma \operatorname{STr}(\sqrt{\operatorname{det}(Q)} \\
& \left.\times \sqrt{-\operatorname{det}\left(\eta_{a b}+\lambda^{2} g_{Y M}^{2} D_{a} X^{i}\left(Q^{-1}\right)^{i j} D_{b} X^{j}+\lambda F_{a b}\right)}\right),
\end{aligned}
$$

where the matrix $Q^{i j}$ is

$$
Q^{i j}=I \delta^{i j}+i \lambda g_{Y M}^{2}\left[X^{i}, X^{j}\right]
$$

where $\lambda \equiv 2 \pi \alpha^{\prime}$. Here the transverse scalars in [11] are normalized as $\Phi^{i}=g_{Y M} \lambda X^{i}$. The trace in the action is completely symmetric between all matrices $F_{a b}, D X^{i},\left[X^{i}, X^{j}\right]$. The D2-brane tension is $T_{2}=1 /(2 \pi)^{2} \ell_{s}^{3} g_{s}$. The 3 -dimensional Yang-Mills coupling constant is related to the tension of D2-brane as $\lambda^{2} T_{2}=1 / g_{Y M}^{2}$.

An extension of the above action to the action of $\mathrm{N} \mathrm{M2-branes} \mathrm{has} \mathrm{been} \mathrm{proposed} \mathrm{in}$ [12]2. This action is given as

$$
\begin{aligned}
& -T_{2} \operatorname{STr}\left(\sqrt{-\operatorname{det}\left(\eta_{a b}+\frac{1}{T_{2}} \tilde{D}_{a} X^{I}\left(\tilde{Q}^{-1}\right)^{(I J)} \tilde{D}_{b} X^{J}\right)}(\operatorname{det}(\tilde{Q}))^{1 / 4}\right)+\operatorname{Tr}\left(\frac{1}{2} \epsilon^{a b c} B_{a} F_{b c}\right)(7) \\
& +\left(\partial_{a} X_{-}^{I}-\operatorname{Tr}\left(B_{a} X^{I}\right)\right) \partial^{a} X_{+}^{I}-\operatorname{Tr}\left(\frac{X_{+} \cdot X}{X_{+}^{2}} \hat{D}_{a} X^{I} \partial^{a} X_{+}^{I}-\frac{1}{2}\left(\frac{X_{+} \cdot X}{X_{+}^{2}}\right)^{2} \partial_{a} X_{+}^{I} \partial^{a} X_{+}^{I}\right)
\end{aligned}
$$

where $X_{+}^{2}=X_{+}^{I} X_{+}^{I}$ and

$$
\begin{aligned}
\tilde{D}_{a} X^{I} & =\hat{D}_{a} X^{I}-\left(\frac{X_{+} \cdot X}{X_{+}^{2}}\right) \partial_{a} X_{+}^{I} \\
\tilde{Q}^{I J} & =s^{I J}+\left(\frac{X_{+}^{I} X_{+}^{J}}{X_{+}^{2}}\right)(\operatorname{det}(s)-1), \quad s^{I J}=\delta^{I J}+\frac{i}{\sqrt{T_{2}}} \frac{X_{+}^{K} M^{I J K}}{\sqrt{X_{+}^{2}}}
\end{aligned}
$$

The M2-brane tension is $T_{2}=1 /(2 \pi)^{2} \ell_{p}^{3}$. It is shown in [12] that this BF type nonlinear Lagrangian reduces to (11) at low energy. Since only the symmetric part of matrix $\tilde{Q}^{-1}$ appears in the action, it includes only terms with even number of $M^{I J K}$.

In this paper we would like to modify this action to includes terms with even and odd number of $M^{I J K}$. To this end, we back to the original action (6) from which the above action has been found. The matrix $\left(Q^{-1}\right)^{i j}$ in the D2-branes action has the following expansion:

$$
\begin{aligned}
\left(Q^{-1}\right)^{i j}= & \delta^{i j}-i \lambda g_{Y M}^{2}\left[X^{i}, X^{j}\right]+\left(i \lambda g_{Y M}^{2}\right)^{2}\left[X^{i}, X^{k}\right]\left[X^{k}, X^{j}\right] \\
& -\left(i \lambda g_{Y M}^{2}\right)^{3}\left[X^{i}, X^{k}\right]\left[X^{k}, X^{l}\right]\left[X^{l}, X^{j}\right]+\cdots
\end{aligned}
$$

\footnotetext{
${ }^{2}$ See 13 for nonlinear action of M2-brane in abelian case.
} 
Using the fact that all brackets appear in symmetric form in the action, one can easily check that all terms with even number of brackets are symmetric in $i, j$ and all terms with odd number of brackets are antisymmetric. It can be written as $\left(Q^{-1}\right)^{i j}=R^{i j}+\lambda P^{i j}$ where the symmetric matrix $R_{i j}$ and antisymmetric matrix $P_{i j}$ are

$$
\begin{aligned}
R^{i j} & =\left(q^{-1}\right)^{i j}, \quad P^{i j}=\left(-i g_{Y M}^{2}\left[X^{i}, X^{n}\right]\right)\left(q^{-1}\right)^{n j} \\
q^{i j} & =\delta^{i j}+\lambda^{2} g_{Y M}^{4}\left[X^{i}, X^{k}\right]\left[X^{k}, X^{j}\right]
\end{aligned}
$$

Note that $1 / \operatorname{det}(R)=\operatorname{det}(q)=(\operatorname{det}(Q))^{2}$.

Both $R^{i j}$ and $P^{i j}$ contributes to action (6). For example, if one considers the $\delta^{i j}$ term of $R_{i j}$, then it is obvious that its contribution is not zero. To see that the antisymmetric matrix $P^{i j}$ has contribution, consider for instance $\left[X^{i}, X^{j}\right]$ term of $P^{i j}$. The expansion of the square root in action (6) has among other things the following term:

$\operatorname{STr}\left(D_{a} X^{i}\left[X^{i}, X^{j}\right] D_{b} X^{j} F_{b a}\right)=\frac{1}{2}\left(\operatorname{Tr}\left(D_{a} X^{i}\left[X^{i}, X^{j}\right] D_{b} X^{j}\right)+\operatorname{Tr}\left(D_{a} X^{i} D_{b} X^{j}\left[X^{i}, X^{j}\right]\right)\right) F_{b a}$

where we have assumed on the right hand side that $F_{a b}$ is abelian. Using the fact that $F_{a b}=-F_{b a}$, one observes that both terms on the right hand side are equal. Hence, $P_{i j}$ has non-vanishing contribution to the action. The symmetric trace makes the matrix $\eta_{a b}+$ $\lambda^{2} g_{Y M}^{2} D_{a} X^{i} R^{i j} D_{b} X^{j}$ in the action to be symmetric and matrix $\lambda^{2} g_{Y M}^{2} D_{a} X^{i} P^{i j} D_{b} X^{j}$ to be antisymmetric.

Now we use the following duality transformation [12]:

$$
-T_{2} \sqrt{-\phi \operatorname{det}\left(g_{a b}+\lambda F_{a b}\right)} \rightarrow-T_{2} \sqrt{-\phi \operatorname{det}\left(g_{a b}+\frac{g_{Y M}^{2}}{T_{2}} \frac{B_{a} B_{b}}{\phi}\right)}+\frac{1}{2} \epsilon^{a b c} B_{a} F_{b c}
$$

for any scalar $\phi$, any symmetric matrix $g_{a b}$ and any antisymmetric matrix $F_{a b}$. The symmetric trace prescription allows us to use the matrix extension of the above identity in which the STr appears in both sides.

Using the above duality transformation, the action (6) can be written in the following form:

$$
\begin{aligned}
S^{D_{2}}= & -T_{2} \int d^{3} \sigma \operatorname{STr}(\sqrt{\operatorname{det}(Q)} \\
& \left.\times \sqrt{-\operatorname{det}\left(\eta_{a b}+\frac{1}{T_{2}} D_{a} X^{i} R^{i j} D_{b} X^{j}+\frac{g_{Y M}^{2}}{T_{2}} \frac{B_{a} B_{b}}{\operatorname{det}(Q)}\right)}\right) \\
& +\frac{1}{2} \int d^{3} \sigma \operatorname{STr}\left(\epsilon^{a b c} B_{a}\left(F_{b c}+\frac{1}{T_{2}} D_{b} X^{i} P^{i j} D_{c} X^{j}\right)\right)
\end{aligned}
$$

where $B_{a}$ is a matrix in the adjoint representation of $U(N)$. 
Using the prescription given in [8], one expects that M2-branes effective action should be reduced to the D2-branes action when $X_{+}^{I}$ takes a large expectation value. Following [12, the M2-branes extension of (12) should have $S O(8)$ invariant term $\tilde{D}_{a} X^{I} \tilde{R}^{I J} \tilde{D}_{b} X^{J}$ where $\tilde{D}_{a} X^{I}$ and $\tilde{R}_{I J}$ should be defined to be invariant under the $B_{a}$ gauge transformation (3) and when $X_{+}^{I}=v \delta^{I 10}$ where $v=g_{Y M}$, they should satisfy the boundary condition [12]:

$$
\tilde{D}_{a} X^{I} \tilde{R}^{I J} \tilde{D}_{b} X^{J} \rightarrow D_{a} X^{i} R^{i j} D_{b} X^{j}+v^{2} \frac{B_{a} B_{b}}{\operatorname{det}(Q)}
$$

This makes $\tilde{D}_{a} X^{I}$ to be defined as in (8) , and the boundary value of $\tilde{R}_{I J}$ to be [12]

$$
\tilde{R}^{i j}=R^{i j} \quad, \quad \tilde{R}^{i 10}=\tilde{R}^{10 i}=0 \quad, \quad \tilde{R}^{1010}=\frac{1}{\operatorname{det}(Q)}
$$

An ansatz for $\tilde{R}^{I J}$ which is consistent with the above boundary condition may be

$$
\tilde{R}^{I J}=\left(S^{-1}\right)^{I J}+a X_{+}^{I} X_{+}^{J}
$$

where $a$ is a $S O(8)$ invariant term which can be found from the above boundary condition, and

$$
S^{I J}=\delta^{I J}-\frac{1}{T_{2}} M^{I K M} M^{J K N}\left(\frac{X_{+}^{M} X_{+}^{N}}{X_{+}^{2}}\right)
$$

Assuming that $M^{I J K}$ should appear in symmetric form in the M2-branes action which is inherited from D2-branes action in which $\left[X^{i}, X^{j}\right]$ appears in symmetric form, one observes that matrix $S^{I J}$ is symmetric. This matrix satisfies the boundary $S^{i j}=q^{i j}, S^{i 10}=S^{10 i}=0$ and $S^{1010}=1$. So at the boundary $\operatorname{det}(S)=\operatorname{det}(q)=(\operatorname{det}(Q))^{2}$. Imposing the boundary condition, one finds

$$
\tilde{R}^{I J}=\left(S^{-1}\right)^{I J}+\frac{X_{+}^{I} X_{+}^{J}}{X_{+}^{2}}\left(\frac{1}{\sqrt{\operatorname{det}(S)}}-1\right)
$$

This symmetric matrix has even number of $M^{I J K}$. Note also $\tilde{R}^{I J}=\delta^{I J}+O\left(\frac{1}{T_{2}}\right)$.

The M2-branes action should also have $S O(8)$ invariant term $\tilde{D}_{a} X^{I} \tilde{P}^{I J} \tilde{D}_{b} X^{J}$ where $\tilde{P}^{I J}$ should be defined to satisfy the boundary condition:

$$
\tilde{D}_{a} X^{I} \tilde{P}^{I J} \tilde{D}_{b} X^{J} \rightarrow D_{a} X^{i} P^{i j} D_{b} X^{j}
$$

This fixes $\tilde{P}^{I J}$ to be

$$
\tilde{P}^{I J}=i M^{I K N}\left(S^{-1}\right)^{N J} X_{+}^{K}
$$


This matrix has odd number of $M^{I J L}$. The symmetric trace prescription then makes it to be antisymmetric matrix. The first term in the third line of (12) is invariant under the gauge transformations associated with $B_{a}$ and $A_{a}$ fields. The second term however is not invariant under these gauge transformations. With the above modification, the second term is not yet invariant under the gauge transformation (3) . To make it invariant, we have to also extend $B_{a} X_{+}^{K}$ to $-\tilde{D}_{a} X^{K}$ which is invariant under the gauge transformation (3).

So, a proposal for the extension of action $S^{D_{2}}$ to M2-branes may be given by the following Lagrangian:

$$
\begin{aligned}
L_{1}^{M_{2}=} & -T_{2} \operatorname{STr}\left((\operatorname{det}(S))^{1 / 4} \sqrt{-\operatorname{det}\left(\eta_{a b}+\frac{1}{T_{2}} \tilde{D}_{a} X^{I} \tilde{R}^{I J} \tilde{D}_{b} X^{J}\right)}\right) \\
& +\frac{1}{2} \epsilon^{a b c}\left(\operatorname{Tr}\left(B_{a} F_{b c}\right)-\frac{i}{T_{2}} \operatorname{STr}\left(\tilde{D}_{a} X^{K} \tilde{D}_{b} X^{I} M^{I K N}\left(S^{-1}\right)^{N J} \tilde{D}_{c} X^{J}\right)\right) \\
& +\left(\partial_{a} X_{-}^{I}-\operatorname{Tr}\left(B_{a} X^{I}\right)\right) \partial^{a} X_{+}^{I}-\operatorname{Tr}\left(\frac{X_{+} \cdot X}{X_{+}^{2}} \hat{D}_{a} X^{I} \partial^{a} X_{+}^{I}-\frac{1}{2}\left(\frac{X_{+} \cdot X}{X_{+}^{2}}\right)^{2} \partial_{a} X_{+}^{I} \partial^{a} X_{+}^{I}\right)
\end{aligned}
$$

The symmetric trace in the first two lines is between gauge invariants $\tilde{D}_{a} X^{I}$ and $M^{I J K}$. This action is manifestly invariant under global $S O(8)$ transformation and is invariant under gauge transformation associated with gauge fields $A_{a}$ and $B_{a}$. As has been discussed in [12], the last line should be added to have consistency with the low energy action.

The equation of motion for $X_{-}^{I}$ gives $\partial_{a} \partial^{a} X_{+}^{I}=0$. As in [12], if one of the scalars $X_{+}^{I}$ takes large expectation value, i.e., $X_{+}^{I}=v \delta^{I 10}$, then $\tilde{D}_{a} X^{i}=D_{a} X^{i}$ and $\tilde{D}_{a} X^{10}=$ $D_{a} X^{10}-v B_{a}$. Now fixing the gauge symmetry (3) by setting $X^{10}=0$, one recovers the D2-branes action (12). On the other hand, if the shift symmetry $X_{-}^{I} \rightarrow X_{-}^{I}+c^{I}$ is gauged as in [14, 15] by introducing a new field $C_{a}^{I}$ and writing $\partial_{a} X_{-}^{I}$ as $\partial_{a} X_{-}^{I}-C_{a}^{I}$, then equation of motion for the new field gives $\partial_{a} X_{+}^{I}=0$ which has only constant solution $X_{+}^{I}=v^{I}$. Using the $S O(8)$ symmetry, one can write it as $X_{+}^{I}=v \delta^{I 10}$. Then the above theory would be classically equivalent to the $D_{2} \bar{D}_{2}$ theory (12).

Using the identity 12

$$
M^{I K M} M^{I K N}\left(\frac{X_{+}^{M} X_{+}^{N}}{X_{+}^{2}}\right)=\frac{1}{3} M^{I J K} M^{I J K}
$$

one finds

$$
(\operatorname{det}(S))^{1 / 4}=1-\frac{1}{12 T_{2}} M^{I J K} M^{I J K}+\cdots
$$

So at low energy the action (19) reduces to (11). The action (19) has terms with even and odd number of $M^{I J K}$. The terms with even number of $M^{I J K}$ are those appear in (77). However, the terms with odd number of $M^{I J K}$ which are non-vanishing, i.e.,

$$
\frac{i}{2 T_{2}} \epsilon^{a b c} \operatorname{STr}\left(\tilde{D}_{a} X^{K} \tilde{D}_{b} X^{I} M^{I K N}\left(S^{-1}\right)^{N L} \tilde{D}_{c} X^{J}\right)
$$


are new couplings that are not included in (7).

Another ansatz for $\tilde{R}^{I J}$ which may be consistent with the boundary condition (14) is

$$
\tilde{R}^{I J}=\left(s^{-1}\right)^{I J}
$$

where

$$
s^{I J}=\delta^{I J}+a M^{I K M} M^{J K M}
$$

in which $a$ is a constant. Imposing the boundary condition $\tilde{R}^{i j}=R^{i j}$, one finds $a=$ $-1 /\left(2 T_{2}\right)$. Interestingly, it also satisfies the boundary condition $\left(s^{-1}\right)^{1010}=1 / \operatorname{det}(Q)$. To see this, consider the expansion of $\left(s^{-1}\right)^{I J}$

$$
\left(s^{-1}\right)^{I J}=\delta^{I J}-a(M M)^{I J}+a^{2}(M M M M)^{I J}-a^{3}(M M M M M M)^{I J}+\cdots
$$

where our notation is such that $(M M M M)^{I J}=M^{I K M} M^{N K M} M^{N P Q} M^{J P Q}$. Using the definition of $M^{I J K}$ in (2), one finds that the 1010 component of this matrix is

$$
\left(s^{-1}\right)^{1010}=\frac{1}{1+a M^{10 i j} M^{10 i j}}=\frac{1}{1+\frac{g_{M Y}^{2}}{T_{2}}\left[X^{i}, X^{j}\right]\left[X^{j}, X^{i}\right]}
$$

On the other hand, because of the symmetric trace in the action, one finds $\operatorname{det}(Q)=$ $1+\frac{g_{M Y}^{2}}{T_{2}}\left[X^{i}, X^{j}\right]\left[X^{j}, X^{i}\right]$. At the boundary, one also has the relation $\operatorname{det}(s)=(\operatorname{det}(Q))^{3}$.

So, another proposal for the extension of action $S^{D_{2}}$ to M2-branes may be given by the following Lagrangian:

$$
\begin{aligned}
L_{2}^{M_{2}}= & -T_{2} \operatorname{STr}\left((\operatorname{det}(s))^{1 / 6} \sqrt{-\operatorname{det}\left(\eta_{a b}+\frac{1}{T_{2}} \tilde{D}_{a} X^{I}\left(s^{-1}\right)^{I J} \tilde{D}_{b} X^{J}\right)}\right) \\
& +\frac{1}{2} \epsilon^{a b c}\left(\operatorname{Tr}\left(B_{a} F_{b c}\right)-\frac{i}{T_{2}} \operatorname{STr}\left(\tilde{D}_{a} X^{K} \tilde{D}_{b} X^{I} M^{I K N}\left(s^{-1}\right)^{N J} \tilde{D}_{c} X^{J}\right)\right) \\
& +\left(\partial_{a} X_{-}^{I}-\operatorname{Tr}\left(B_{a} X^{I}\right)\right) \partial^{a} X_{+}^{I}-\operatorname{Tr}\left(\frac{X_{+} \cdot X}{X_{+}^{2}} \hat{D}_{a} X^{I} \partial^{a} X_{+}^{I}-\frac{1}{2}\left(\frac{X_{+} \cdot X}{X_{+}^{2}}\right)^{2} \partial_{a} X_{+}^{I} \partial^{a} X_{+}^{I}\right)
\end{aligned}
$$

In this case also, using the expansion

$$
(\operatorname{det}(s))^{1 / 6}=1-\frac{1}{12 T_{2}} M^{I J K} M^{I J K}+\cdots
$$

one observes that at low energy the above action reduces to (11) as expected. For constant $X_{+}^{I}$, the above action produces the couplings found in [16] at order $1 / T_{2}$. 


\section{References}

[1] J. H. Schwarz, JHEP 0411, 078 (2004) arXiv:hep-th/0411077.

A. Basu and J. A. Harvey, Nucl. Phys. B 713, 136 (2005) arXiv:hep-th/0412310.

[2] J. Bagger and N. Lambert, JHEP 0802, 105 (2008) arXiv:0712.3738.

J. Bagger and N. Lambert, Phys. Rev. D 77, 065008 (2008) arXiv:0711.0955.

J. Bagger and N. Lambert, Phys. Rev. D 75, 045020 (2007) arXiv:hep-th/0611108.

[3] A. Gustavsson, arXiv:0709.1260.

A. Gustavsson, JHEP 0804, 083 (2008) arXiv:0802.3456.

[4] N. Lambert and D. Tong, arXiv:0804.1114. J. Distler, S. Mukhi, C. Papageorgakis and M. Van Raamsdonk, JHEP 0805, 038 (2008) arXiv:0804.1256. C. Krishnan and C. Maccaferri, JHEP bf 0807, 005 (2008) [arXiv:0805.3125 [hep-th]].

[5] O. Aharony, O. Bergman, D. L. Jafferis and J. Maldacena, arXiv:0806.1218.

[6] J. Gomis, G. Milanesi and J. G. Russo, JHEP 0806, 075 (2008) arXiv:0805.1012. S. Benvenuti, D. Rodriguez-Gomez, E. Tonni and H. Verlinde, arXiv:0805.1087. P. M. Ho, Y. Imamura and Y. Matsuo, JHEP 0807, 003 (2008) arXiv:0805.1202.

[7] S. Cecotti and A. Sen, arXiv:0806.1990 [hep-th]; P. de Medeiros, J. M. FigueroaO'Farrill and E. Mendez-Escobar, arXiv:0806.3242 [hep-th]; M. Ali-Akbari, M. M. Sheikh-Jabbari and J. Simon, arXiv:0807.1570 [hep-th]; H. Verlinde, arXiv:0807.2121 [hep-th].

[8] S. Mukhi and C. Papageorgakis, JHEP 0805, 085 (2008) [arXiv:0803.3218 [hep-th]].

[9] H. Nicolai and H. Samtleben, Nucl. Phys. B 668, 167 (2003) arXiv:hep-th/0303213; B. de Wit, I. Herger and H. Samtleben, Nucl. Phys. B 671, 175 (2003) arXiv:hep-th/0307006; B. de Wit, H. Nicolai and H. Samtleben, arXiv:hep-th/0403014.

[10] A. A. Tseytlin, Nucl. Phys. B 501, 41 (1997) arXiv:hep-th/9701125.

[11] R. C. Myers, JHEP 9912, 022 (1999) arXiv:hep-th/9910053.

[12] R. Iengo and J. G. Russo, arXiv:0808.2473 [hep-th].

[13] J. Kluson, arXiv:0807.4054 [hep-th].

[14] M. A. Bandres, A. E. Lipstein and J. H. Schwarz, arXiv:0806.0054 .

J. Gomis, D. Rodriguez-Gomez, M. Van Raamsdonk and H. Verlinde, arXiv:0806.0738. 
[15] B. Ezhuthachan, S. Mukhi and C. Papageorgakis, JHEP 0807, 041 (2008) arXiv:0806.1639 [hep-th]];

[16] M. Alishahiha and S. Mukhi, arXiv:0808.3067 [hep-th]. 